\title{
Review: thrombolysis increases short term death and intracranial haemorrhage but decreases long term death or dependence
}

\author{
Wardlaw JM. Overview of Cochrane thrombolysis meta-analysis. Neurology 2001 Sep;57(Suppl 2):S69-76. \\ Wardlaw JM, del Zoppo G, Yamaguchi T. Thrombolysis for acute ischaemic stroke. Cochrane Database Syst Rev \\ 2001;(4):CD000213 (latest version 24 Jul 2001).
QUESTION: In patients with acute ischaemic stroke, is thrombolytic treatment safe and effective for reducing mortality and intracranial haemorrhage, and for improving functional outcome?

\section{Data sources}

Studies were identified by searching the Cochrane Specialised Register of Controlled Trials and EMBASE/ Excerpta Medica, hand searching neurological and stroke journals, writing to 321 pharmaceutical companies for information, and reviewing meeting abstracts and conference proceedings.

\section{Study selection}

Studies were selected if they were randomised controlled trials that compared thrombolytic drugs with placebo or control treatment in patients with definite acute ischaemic stroke. Trials were excluded if the treatment or control group received another active treatment not factored into the randomisation.

\section{Data extraction}

Data were extracted on patients, drug dose, administration route, duration of treatment, stroke type, results of
Scotland, UK.

jmw@skull.dcn.ed.ac.uk. computed tomography, and duration of follow up. Outcomes were all-cause mortality 7 to 10 days after treatment, symptomatic or fatal intracranial haemorrhage, all-cause mortality at long term follow up, and poor functional outcome (death or dependence measured by the Rankin or Barthel scales). Study quality was assessed on randomisation method, blinding of drug administration, and blinding of outcome assessment.

\section{Main results}

17 trials (5144 patients) were included. Thrombolysis comprised intravenous (IV) streptokinase (4 trials), IV recombinant tissue plasminogen activator (rt-PA) (8 trials), IV urokinase (3 trials), and intra-arterial recombinant pro-urokinase ( 2 trials). About half the trials studied rt-PA. Most patients were treated within 6 hours of stroke. Thrombolysis increased all-cause mortality $(p<0.001)$ and symptomatic or fatal intracranial haemorrhage $(\mathrm{p}<0.001)$ within 7 to 10 days of treatment (table). Heterogeneity existed among trials of all-cause mortality at the end of follow up (1 to $6 \mathrm{mo}$ ), even when the analysis was restricted to trials of rt-PA and showed a non-statistically significant increase in death in patients receiving thrombolysis (table). Death or dependence was decreased at the end of follow up in patients who received thrombolysis $(\mathrm{p}=0.003)$ (table).

\section{Conclusion}

In patients with acute ischaemic stroke, thrombolytic treatment increases short term mortality and symptomatic or fatal intracranial haemorrhage, but decreases longer term death or dependence.

${ }^{*} \mathrm{FIH}=$ fatal intracranial haemorrhage; $\mathrm{NS}=$ not significant; $\mathrm{SIH}=$ symptomatic intracranial haemorrhage. Other abbreviations defined in glossary; RRI, RRR, NNH, NNT, and Cl calculated from data in article.

†Random effects model used because heterogeneity existed.

\section{COMMENTARY}

The review by Wardlaw is an update of the Cochrane review of thrombolysis for acute ischaemic stroke. Like all meta-analyses, it necessarily includes different forms, administration rates, and doses of treatment. Although the overview analysis is important, the real interest is in the subanalyses.

t-PA is the most commonly used thrombolytic agent for ischaemic stroke and is usually given intravenously within 3 hours of stroke onset. For this subanalysis, the number of patients saved from death or dependence per 1000 treated patients was 140 (95\% CI 77 to 203) and from death alone was 12 (CI 61 fewer to 38 more). In biological terms, this medicine is powerful and one would surmise that it should have a substantial effect on the burden of stroke. Unfortunately, it has not. Despite encouraging phase IV data, the number of patients to whom this treatment has become available is small, probably $1 \%$ to $2 \%$ of those with ischaemic strokes.

The meta-analysis gives us some reason to hope that this level of penetration may be improved. When time windows of up to 6 hours after stroke were considered in patients given IV t-PA, 57 (CI 20 to 93) fewer patients died or were dependent per 1000 treated. Fortunately, only a modest trend existed toward increased death alone (18 per 1000 treated). Why then is t-PA not licensed for a 6 hour time window?

Only 1 pivotal trial exists in which t-PA has been unequivocally beneficial. ${ }^{1}$ The time window used was 3 hours. All other trials have shown trends toward a beneficial outcome, but they did not satisfy the 0.05 judge. Furthermore, even the modest increase in mortality, naturally, concerns many.

More patients need to be entered into randomised trials, particularly with treatment after 3 hours or including other circumstances in which risk and benefit are uncertain. Magnetic resonance imaging may also be useful in selecting appropriate patients. ${ }^{2}$ 